\title{
Robust decentralized static output feedback *
}

\author{
Dennis S. BERNSTEIN \\ Harris Corporation, Government Aerospace Systems Division, MS 22/4848, Melbourne, FL 32902, U.S.A.
}

\author{
Wassim M. HADDAD \\ Department of Mechanical and Aerospace Engineering, Florida Institute of Technology, Melbourne, FL 32901, U.S.A.
}

Received 18 March 1988

Revised 29 October 1988

\begin{abstract}
Sufficient conditions are developed for characterizing robust decentralized static output feedback controllers. The approach involves deriving necessary conditions for minimizing a bound on closed-loop performance over a specified range of uncertain parameters. The effect of plant parameter variations on the closed-loop covariance is overbounded by means of a modified Lyapunov equation whose solutions are guaranteed to provide robust stability and performance.
\end{abstract}

Keywords: Robust control; guaranteed bounds; optimal; structured uncertainty; real parameters.

\section{Introduction}

Because of implementation constraints, cost, and reliability considerations, a decentralized controller architecture is often desirable for controlling large scale systems. Furthermore, such controllers must be robust to variations in plant parameters. The present paper addresses both of these concerns within the context of a robust decentralized theory for continuous-time static (i.e., proportional) controllers.

The approach to controller design considered herein involves optimizing closed-loop performance with respect to the feedback gains. This approach to static output feedback was developed for centralized controllers in $[9,11]$ and for decentralized controllers in [12]. An interesting feature of $[11,12]$ is the recognition of an oblique projection (idempotent matrix) which allows the necessary conditions to be written concisely in terms of a modified algebraic Riccati equation. When the problem is specialized to full-state feedback, the projection becomes the identity and the modified Riccati equation coincides with the standard Riccati equation of LQR theory. It should be pointed out that this oblique projection is distinct from the oblique projection arising in dynamic compensation [5].

The present paper goes beyond [12] by deriving sufficient conditions for characterizing decentralized static controllers which guarantee robust stability and performance with respect to variations in the plant parameters. Although plant disturbances are represented in the usual stochastic manner by means of additive white noise, uncertainty in the plant dynamics is modeled deterministically by means of constant structured parameter variations within bounded sets. Thus, for example, the dynamics matrix $A$ is replaced by $A+\sum_{k=1}^{p} \sigma_{k} A_{k}$, where $\sigma_{k}$ is a constant uncertain parameter assumed only to lie within the interval $\left[-\alpha_{k}, \alpha_{k}\right]$ but otherwise unknown, and $A_{k}$ is a fixed matrix denoting the structure of the uncertain parameter $\sigma_{k}$ as it appears in the nominal dynamics matrix $A$. The closed-loop performance is defined to be the worst-case value over the class of parameter uncertainties of a quadratic criterion averaged over the disturbance statistics.

Since the closed-loop performance can be written in terms of the second-moment matrix, a performance bound over the class of uncertain parameters can be obtained by bounding the state covariance. The key to bounding the state covariance is to replace the usual Lyapunov equation for the second-moment matrix by

* Supported in part by the Air Force Office of Scientific Research under contracts F49620-86-C-0002 and F49620-86-C-0038. 
a modified Lyapunov equation. The modified Lyapunov equation involves additional terms which essentially serve to construct a quadratic Lyapunov function guaranteeing robust stability. For details see $[1,2]$.

Having bounded the state covariance over the class of parameter uncertainties, the worst-case performance can thus be bounded in terms of the solution to the modified Lyapunov equation. Viewing the performance bound as an auxiliary cost thus leads to the Auxiliary Minimization Problem: Minimize the performance bound while satisfying the modified Lyapunov equation. The principal feature of the auxiliary problem is that necessary conditions for minimizing the performance bound now serve as sufficient conditions for robust performance in the original problem. Philosophically, the overall approach of control design for a performance bound is related to guaranteed cost control [4]. We note, however, that the bound utilized in [4] is nondifferentiable, which precludes the approach of the present paper.

A further extension of [12] considered in the present paper involves the types of feedback loops considered. While the usual approach to static output feedback is restricted to nonnoisy measurements and weighted controls, we also include the dual problem which involves feeding back noisy measurements to unweighted controls. This situation leads to an additional projection which is dual to the projection discussed in [11,12]. The inclusion of the dual case now leads to a pair of modified Riccati equations coupled by both the uncertainty bounds and the oblique projections.

\section{Notation and definitions}

$\mathbb{R}, \mathbb{R}^{r \times s}, \mathbb{R}^{r}, \mathbb{E} \quad$ real numbers, $r \times s$ real numbers, $\mathbb{R}^{r \times 1}$, expectation.

$I_{r},()^{\mathrm{T}} \quad r \times r$ identity, transpose.

$\oplus, \otimes$

$\mathbb{S}^{r}$

$\mathbb{N}^{r}$

$\mathbb{P}^{r}$

$Z_{1} \leqslant Z_{2}$

Kronecker sum, Kronecker product [3].

$r \times r$ symmetric matrices.

$r \times r$ symmetric nonnegative-definite matrices.

$r \times r$ symmetric positive-definite matrices.

$Z_{1}<Z_{2}$

$Z_{2}-Z_{1} \in \mathbb{N}^{r}, Z_{1}, Z_{2} \in \mathbb{S}^{r}$.

$Z_{2}-Z_{1} \in \mathbb{P}^{r}, Z_{1}, Z_{2} \in \mathbb{S}^{r}$.

Asymptotically

stable matrix

$n, r, s, p$

$i, j, k$

$m_{i}, \hat{l}_{i}$

$\hat{m}_{j}, l_{j}$

$x$

$u_{i}, \hat{y}_{i}$

$\hat{u}_{j}, y_{j}$

$A, \Delta A$

$B_{i}, \Delta B_{i} ; \hat{C}_{i}$

$\hat{B}_{j} ; C_{j}, \Delta C_{j}$

$A_{k}$

$B_{i k}$

$C_{j k}$

$D_{c i}$

$E_{\text {cj }}$

$\alpha$

$A_{\alpha}$

$\alpha_{k}$

$\gamma_{k}$

matrix with eigenvalues in open left half plane.

positive integers.

indices, $i=1, \ldots, r, j=1, \ldots, s, k=1, \ldots, p$.

positive integers, $i=1, \ldots, r$.

positive integers, $j=1, \ldots, s$.

$n$-dimensional vector.

$m_{i}, \hat{l}_{i}$-dimensional vectors, $i=1, \ldots, r$.

$\hat{m}_{j}, l$-dimensional vectors, $j=1, \ldots, s$.

$n \times n$ matrices.

$n \times m_{i}$ matrices; $\hat{l}_{i} \times n$ matrices, $i=1, \ldots, r$.

$n \times \hat{m}_{j}$ matrices; $l_{j} \times n$ matrices, $j=1, \ldots, s$.

$n \times n$ matrices, $k=1, \ldots, p$.

$n \times m_{i}$ matrices, $i=1, \ldots, r, k=1, \ldots, p$.

$l_{j} \times n$ matrices, $j=1, \ldots, s, k=1, \ldots, p$.

$m_{i} \times \hat{l}_{i}$ matrices, $i=1, \ldots, r$.

$\hat{m}_{j} \times l$, matrices, $j=1, \ldots, s$.

positive number.

$A+\frac{1}{2} \alpha I_{n}$.

positive number, $k=1, \ldots, p$.

$\alpha_{k}^{2} / \alpha, k=1, \ldots, p$.

$\sigma_{k}$

real number, $k=1, \ldots, p$. 
$w_{0}(t), w_{j}(t)$

$V_{0}, V_{j}$

$V_{0 j}$

$R_{0}, R_{i}$

$R_{0 i}$

$\tilde{A}, \tilde{A}_{\alpha}$

$\Delta \tilde{A}$

$\tilde{w}(t)$

$\tilde{R}$

$\tilde{V}$ $n$-dimensional, $l_{j}$-dimensional white noise, $j=1, \ldots, s$.

intensities of $w_{0}, w_{j} ; V_{0} \in \mathbb{N}^{n}, V_{j} \in \mathbb{P}^{l_{j}}, j=1, \ldots, s$.

$n \times l_{j}$ cross intensity of $w_{0}, w_{j}, j=1, \ldots, s$.

state and control weightings; $R_{0} \in \mathbb{N}^{n}, R_{i} \in \mathbb{P}^{m_{i}}, i=1, \ldots, r$.

$n \times m_{i}$ cross weighting; $R_{0}-R_{0 i} R_{i}^{-1} R_{0 i}^{\mathrm{T}} \geqslant 0, i=1, \ldots, r$.

$A+\sum_{i=1}^{r} B_{i} D_{c i} \hat{C}_{i}+\sum_{j=1}^{s} \hat{B}_{j} E_{c j} C_{j}, \tilde{A}+\frac{1}{2} \alpha I_{n}$.

$\Delta A+\sum_{i=1}^{r} \Delta B_{i} D_{c i} \hat{C}_{i}+\sum_{j=1}^{s} \hat{B}_{j} E_{c j} \Delta C_{j}$.

$w_{0}(t)+\sum_{j=1}^{s} B_{j} E_{c j} w_{j}(t)$.

$R_{0}+\sum_{i=1}^{r}\left[R_{0 i} D_{c i} \hat{C}_{i}+\hat{C}_{i}^{\mathrm{T}} D_{c i}^{\mathrm{T}} R_{0 i}^{\mathrm{T}}+\hat{C}_{i}^{\mathrm{T}} D_{c i}^{\mathrm{T}} R_{i} D_{0 i} \hat{C}_{i}\right]$.

$V_{0}+\sum_{j=1}^{s}\left[V_{0 j} E_{c j}^{\mathrm{T}} \hat{B}_{j}^{\mathrm{T}}+\hat{B}_{j} E_{c j} V_{0 j}^{\mathrm{T}}+\hat{B}_{j} E_{c j} V_{j} E_{c j}^{\mathrm{T}} \hat{B}_{j}^{\mathrm{T}}\right]$.

For arbitrary $n \times n Q, P$ define

$$
\begin{array}{lll}
R_{a i} \triangleq R_{i}+\sum_{k=1}^{p} \gamma_{k} B_{i k}^{\mathrm{T}} P B_{k}, & P_{a i} \triangleq B_{i}^{\mathrm{T} P}+R_{0 i}^{\mathrm{T}}+\sum_{k=1}^{p} \gamma_{k} B_{i k}^{\mathrm{T}} P A_{k}, & i=1, \ldots, r, \\
V_{a j} \triangleq V_{j}+\sum_{k=1}^{p} \gamma_{k} C_{j k} Q C_{j k}^{\mathrm{T}}, & Q_{a j} \triangleq Q C_{j}^{\mathrm{T}}+V_{0 j}+\sum_{k=1}^{p} \gamma_{k} A_{k} Q C_{j k}^{\mathrm{T}}, & j=1, \ldots, s .
\end{array}
$$

\section{Robust Stability and Performance Problem}

In this section we state the Robust Stability and Performance Problem along with related notation for later use. Let

$$
\mathscr{U} \subset \mathbb{R}^{n \times n} \times \mathbb{R}^{n \times m_{1}} \times \cdots \times \mathbb{R}^{n \times m_{r}} \times \mathbb{R}^{l_{1} \times n} \times \cdots \times \mathbb{R}^{l_{s} \times n}
$$

denote the set of uncertain perturbations $\left(\Delta A, \Delta B_{1}, \ldots, \Delta B_{r}, \Delta C_{1}, \ldots, \Delta C_{s}\right)$ of the nominal system matrices $A, B_{1}, \ldots, B_{r}, C_{1}, \ldots, C_{s}$.

Robust Stability and Performance Problem. For the $n$ th-order plant

$$
\dot{x}(t)=(A+\Delta A) x(t)+\sum_{i=1}^{r}\left(B_{i}+\Delta B_{i}\right) u_{i}(t)+\sum_{j=1}^{s} \hat{B}_{j} \hat{u}_{j}(t)+w_{0}(t), \quad t \in[0, \infty),
$$

with nonnoisy and noisy measurements

$$
\begin{aligned}
& \hat{y}_{i}(t)=\hat{C}_{i} x(t), \quad i=1, \ldots, r, \\
& y_{j}(t)=\left(C_{j}+\Delta C_{j}\right) x(t)+w_{j}(t), \quad j=1, \ldots, s,
\end{aligned}
$$

determine a decentralized static output feedback controller

$$
\begin{aligned}
& u_{i}(t)=D_{c i} \hat{y}_{i}(t), \quad i=1, \ldots, r, \\
& \hat{u}_{j}(t)=E_{c j} y_{j}(t), \quad j=1, \ldots, s,
\end{aligned}
$$

such that the closed-loop system (3.1)-(3.5) is asymptotically stable for all variations in $\mathscr{U}$ and such that the performance criterion

$$
\begin{aligned}
& J\left(D_{c 1}, \ldots, D_{c r}, E_{c 1}, \ldots, E_{c s}\right) \\
& \quad \triangleq \sup _{\mathscr{Q}} \limsup _{t \rightarrow \infty} \mathbb{E}\left[x^{\mathrm{T}}(t) R_{0} x(t)+2 \sum_{i=1}^{r} x^{\mathrm{T}}(t) R_{0 i} u_{i}(t)+\sum_{i=1}^{r} u_{i}^{\mathrm{T}}(t) R_{i} u_{i}(t)\right]
\end{aligned}
$$

is minimized. 
For each controller $\left(D_{c 1}, \ldots, D_{c r}, E_{c 1}, \ldots, E_{c s}\right)$ and variation in $\mathscr{U}$, the closed-loop system (3.1)-(3.5) is given by

$$
\dot{x}(t)=(\tilde{A}+\Delta \tilde{A}) x(t)+\tilde{w}(t), \quad t \in[0, \infty),
$$

where $\tilde{w}(t)$ is white noise with intensity $\tilde{V} \in \mathbb{N}^{n}$.

Remark 3.1. The controller architecture is quite general in that it includes two distinctly different types of decentralized loops. The first type, indexed by $i=1, \ldots, r$, involves feeding back nonnoisy measurements to weighted controls. This is the standard setting in the optimal output-feedback literature $[9,11,12]$. In addition, we include the dual situation, indexed by $j=1, \ldots, s$, which involves feeding back noisy measurements to unweighted controls. The case in which only one type of loop is present can be formally recovered from our results by ignoring $B_{i}$ and $\hat{C}_{i}$ or $\hat{B}_{j}$ and $C_{j}$ as required. We note that in order for (3.6) to be finite noisy measurements cannot be fed back to weighted controls via static control, while feeding back nonnoisy measurements to unweighted controls is a singular problem.

Remark 3.2. The problem statement is restrictive in the sense that uncertainties in both the control and observation matrices are not permitted within the same feedback loop. Although it is indeed possible to permit such simultaneous uncertainties, the development is considerably more complex and hence is not treated here.

Remark 3.3. The cost functional (3.6) is identical to the LQG criterion (usually stated in terms of an averaged integral) with the exception of the supremum for evaluating worst case over $\mathscr{U}$.

\section{Sufficient conditions for robust stability and performance}

In practice, steady-state performance is only of interest when the closed-loop system (3.7) is stable over the uncertainty set $\mathscr{U}$. The following result, which expresses the performance (3.6) in terms of the state covariance, is immediate.

Lemma 4.1. Let $\left(D_{c 1}, \ldots, D_{c r}, E_{c 1}, \ldots, E_{c s}\right)$ be given and suppose the closed-loop system (3.7) is stable for all variations in $\mathscr{U}$. Then

$$
J\left(D_{c 1}, \ldots, D_{c r}, E_{c 1}, \ldots, E_{c s}\right)=\sup _{\mathscr{Q}} \operatorname{tr} Q_{\Delta \tilde{A}} \tilde{R},
$$

where $Q_{\Delta \tilde{A}} \triangleq \lim _{t \rightarrow \infty} \mathbb{E}\left[x(t) x^{\mathrm{T}}(t)\right] \in \mathbb{N}^{n}$ is the unique solution to

$$
0=(\tilde{A}+\Delta \tilde{A}) Q_{\Delta \tilde{A}}+Q_{\Delta \tilde{A}}(\tilde{A}+\Delta \tilde{A})^{\mathrm{T}}+\tilde{V} .
$$

Remark 4.1. When $\mathscr{U}$ is compact, sup in (4.1) can be replaced by max.

We now seek an upper bound for $J\left(D_{c 1}, \ldots, D_{c r}, E_{c 1}, \ldots, E_{c s}\right)$. The key step is to modify the Lyapunov equation (4.2) to include a bounding function $\Omega$. This technique allows us to obtain robust stability as a consequence of robust performance.

Theorem 4.1. Let $\Omega: \mathbb{R}^{m_{1} \times \hat{l}_{1}} \times \cdots \times \mathbb{R}^{m_{r} \times \hat{l}_{r}} \times \mathbb{R}^{\hat{m}_{1} \times l_{1}} \times \cdots \times \mathbb{R}^{\hat{m}_{s} \times l_{s}} \times \mathbb{N}^{n} \rightarrow \mathbb{S}^{n}$ be such that

$$
\begin{aligned}
& \Delta \tilde{A} Q+Q \Delta \tilde{A}^{\mathrm{T}} \leq \Omega\left(D_{c 1}, \ldots, D_{c r}, E_{c 1}, \ldots, E_{c s}, Q\right), \\
& \left(\Delta A, \Delta B_{1}, \ldots, \Delta B_{r}, \Delta C_{1}, \ldots, \Delta C_{s}\right) \in \mathscr{U}, \\
& \left(D_{c 1}, \ldots, D_{c r}, E_{c 1}, \ldots, E_{c s}, Q\right) \in \mathbb{R}^{m_{1} \times \hat{l}_{1}} \times \cdots \times \mathbb{R}^{m_{r} \times \hat{i}_{r}} \times \mathbb{R}^{\hat{m}_{1} \times l_{1}} \times \cdots \times \mathbb{R}^{\hat{m}_{s} \times l_{s}} \times \mathbb{N}^{n} .
\end{aligned}
$$


Furthermore, for given $\left(D_{c 1}, \ldots, D_{c r}, E_{c 1}, \ldots, E_{c s}\right)$ suppose there exists $Q \in \mathbb{N}^{n}$ satisfying

$$
0=\tilde{A} Q+Q \tilde{A}^{\mathrm{T}}+\Omega\left(D_{c 1}, \ldots, D_{c r}, E_{c 1}, \ldots, E_{c s}, Q\right)+\tilde{V} .
$$

Then the pair $\left(\tilde{A}+\Delta \tilde{A}, \tilde{V}^{1 / 2}\right)$ is stabilizable for all variations in $\mathscr{U}$ if and only if $\tilde{A}+\Delta \tilde{A}$ is asymptotically stable for all variations in $\mathscr{U}$. In this case,

$$
Q_{\Delta \tilde{A}} \leq Q
$$

where $Q_{\Delta \tilde{A}}$ satisfies (4.2), and

$$
J\left(D_{c 1}, \ldots, D_{c r}, E_{c 1}, \ldots, E_{c s}\right) \leq \operatorname{tr} Q \tilde{R} .
$$

Proof. For all variations in $\mathscr{U},(4.4)$ is equivalent to

$$
0=(\tilde{A}+\Delta \tilde{A}) Q+Q(\tilde{A}+\Delta \tilde{A})^{\mathbf{T}}+\Phi\left(D_{c 1}, \ldots, D_{c r}, E_{c 1}, \ldots, E_{c s}, Q, \Delta \tilde{A}\right)+\tilde{V}
$$

where

$$
\Phi\left(D_{c 1}, \ldots, D_{c r}, E_{c 1}, \ldots, E_{c s}, Q, \Delta \tilde{A}\right) \triangleq \Omega\left(D_{c 1}, \ldots, D_{c r}, E_{c 1}, \ldots, E_{c s}, Q\right)-\left(\Delta \tilde{A} Q+Q \Delta \tilde{A}^{\mathrm{T}}\right) .
$$

Note that by $(4.3), \Phi(\cdot) \geq 0$ for all variations in $\mathscr{U}$. If $\left(\tilde{A}+\Delta \tilde{A}, \tilde{V}^{1 / 2}\right)$ is stabilizable for all variations in $\mathscr{Q}$, it follows from Theorem 3.6 of [14] that $\left(\tilde{A}+\Delta \tilde{A},\left[\tilde{V}+\Phi\left(D_{c 1}, \ldots, D_{c r}, E_{c 1}, \ldots, E_{c s}, Q, \Delta \tilde{A}\right)\right]^{1 / 2}\right)$ is stabilizable for all variations in $\mathscr{U}$. Hence Lemma 12.2 of [14] implies that $\tilde{A}+\Delta \tilde{A}$ is asymptotically stable for all variations in $\mathscr{U}$. The converse is immediate. Next, substracting (4.2) from (4.7) yields

$$
0=(\tilde{A}+\Delta \tilde{A})\left(Q-Q_{\Delta \tilde{A}}\right)+\left(Q-Q_{\Delta \tilde{A}}\right)(\tilde{A}+\Delta \tilde{A})^{\mathrm{T}}+\Phi\left(D_{c 1}, \ldots, D_{c r}, E_{c 1}, \ldots, E_{c s}, Q, \Delta \tilde{A}\right),
$$

or, equivalently, (since $\tilde{A}+\Delta \tilde{A}$ is asymptotically stable)

$$
Q-Q_{\Delta \tilde{A}}=\int_{0}^{\infty} \mathrm{e}^{(\tilde{A}+\Delta \tilde{A}) t} \Phi\left(D_{c 1}, \ldots, D_{c r}, E_{c 1}, \ldots, E_{c s}, Q, \Delta \tilde{A}\right) \mathrm{e}^{(\tilde{A}+\Delta \tilde{A})^{\mathrm{T}} t} \mathrm{~d} t \geq 0,
$$

which implies (4.5). Finally, (4.5) and (4.1) yield (4.6).

Remark 4.2. If $\tilde{V}$ is positive definite then the stabilizability hypothesis of Theorem 4.1 is automatically satisfied for all variations in $\mathscr{U}$.

\section{Uncertainty structure and the quadratic Lyapunov bound}

The uncertainty set $\mathscr{U}$ is assumed to be of the form

$$
\begin{gathered}
\mathscr{Q}=\left\{\left(\Delta A, \Delta B_{1}, \ldots, \Delta B_{r}, \Delta C_{1}, \ldots, \Delta C_{s}\right):\right. \\
\Delta A=\sum_{k=1}^{p} \sigma_{k} A_{k}, \Delta B_{i}=\sum_{k=1}^{p} \sigma_{k} B_{i k}, i=1, \ldots, r, \\
\left.\Delta C_{j}=\sum_{k=1}^{p} \sigma_{k} C_{j k}, j=1, \ldots, s, \sum_{k=1}^{p} \sigma_{k}^{2} / \alpha_{k}^{2} \leq 1\right\},
\end{gathered}
$$

where, for $k=1, \ldots, p:\left(A_{k}, B_{1 k}, \ldots, B_{r k}, C_{1 k}, \ldots, C_{s k}\right)$ are fixed matrices denoting the structure of the parametric uncertainty; $\alpha_{k}$ is a given uncertainty bound; and $\sigma_{k}$ is an uncertain parameter. Note that the 
uncertain parameters $\sigma_{k}$ are assumed to lie in a specified ellipsoidal region in $\mathbb{R}^{p}$. The closed-loop system (3.7) thus has structured uncertainty of the form

$$
\Delta \tilde{A}=\sum_{k=1}^{p} \sigma_{k} \tilde{A_{k}}
$$

where

$$
\tilde{A_{k}} \triangleq A_{k}+\sum_{i=1}^{r} B_{i k} D_{c i} \hat{C}_{i}+\sum_{j=1}^{s} \hat{B}_{j} E_{c j} C_{j k}, \quad k=1, \ldots, p .
$$

To obtain explicit gain expressions for the feedback gains $\left(D_{c 1}, \ldots, D_{c r}, E_{c 1}, \ldots, E_{c s}\right)$ we assume that, for each $k \in\{1, \ldots, p\}$, at most one of the matrices $B_{1 k}, \ldots, B_{r k}, C_{1 k}, \ldots, C_{s k}$ is nonzero. This assumption requires that uncertainties in the input and output matrices be modeled as uncorrelated. Correlation between $\Delta A$ and $\Delta B_{i}$ and between $\Delta A$ and $\Delta C_{j}$ can be accounted for, however.

Given the structure of $\mathscr{U}$ defined by (5.1), the bound $\Omega$ satisfying (4.3) can now be specified. In the following result [as in (4.3)] $Q$ denotes an arbitrary element of $\mathbb{N}^{n}$, not necessarily a solution to (4.4).

Proposition 5.1. Let $\alpha$ be an arbitrary positive scalar. Then the function

$$
\Omega\left(D_{c 1}, \ldots, D_{c r}, E_{c 1}, \ldots, E_{c s}, Q\right)=\alpha Q+\alpha^{-1} \sum_{k=1}^{p} \alpha_{k}^{2} \tilde{A}_{k} Q \tilde{A}_{k}^{\mathrm{T}}
$$

satisfies (4.3) with $\mathscr{U}$ given by (5.1).

Proof. Note that

$$
\begin{aligned}
0 & \leq \sum_{k=1}^{p}\left[\left(\alpha^{1 / 2} \sigma_{k} / \alpha_{k}\right) I_{n}-\left(\alpha_{k} / \alpha^{1 / 2}\right) \tilde{A}_{k}\right] Q\left[\left(\alpha^{1 / 2} \sigma_{k} / \alpha_{k}\right) I_{n}-\left(\alpha_{k} / \alpha^{1 / 2}\right) \tilde{A}_{k}\right]^{\mathrm{T}} \\
& =\alpha \sum_{k=1}^{p}\left(\sigma_{k}^{2} / \alpha_{k}^{2}\right) Q+\alpha^{-1} \sum_{k=1}^{p} \alpha_{k}^{2} \tilde{A_{k}} Q \tilde{A}_{k}^{\mathrm{T}}-\sum_{k=1}^{p} \sigma_{k}\left(\tilde{A_{k}} Q+Q \tilde{A}_{k}^{\mathrm{T}}\right)
\end{aligned}
$$

which yields (4.3).

Remark 5.1. Note that the bound $\Omega$ given by (5.4) consists of two distinct terms. The first term $\alpha Q$ can be thought of as arising from an exponential time weighting of the cost, or, equivalently, from a uniform right shift of the open-loop dynamics. The second term $\alpha^{-1} \sum_{k=1}^{p} \alpha_{k}^{2} \tilde{A_{k}} Q \tilde{A}_{k}^{\mathrm{T}}$ arises naturally from a multiplicative white noise model [1]. Such interpretations have no bearing on the results obtained here since only the bound $\Omega$ defined by (5.4) is required. Note that the bound is valid for all positive $\alpha$. For further details see [1].

Remark 5.2. The conservatism of the bound (5.4) is difficult to predict for two reasons. First, the overbounding (4.3) holds with respect to the partial ordering of the nonnegative-definite matrices for which no scalar measure of conservatism is available. And, second, the bound (4.3) is required to hold for all feedback gains $\left(D_{c 1}, \ldots, D_{c r}, E_{c 1}, \ldots, E_{c s}\right)$ and nonnegative-definite matrices $Q$. The conservatism will thus depend upon the actual values of $E_{c 1}, \ldots, D_{c r}, E_{c 1}, \ldots, E_{c s}, Q$ determined by solving (4.4).

\section{The Auxiliary Minimization Problem and necessary conditions for optimality}

Rather than minimizing the actual cost (3.6), we shall consider the upper bound (4.6). This leads to the following problem. 
Auxiliary Minimization Problem. Determine $\left(D_{c 1}, \ldots, D_{c r}, E_{c 1}, \ldots, E_{c s}, Q\right)$ which minimizes

$$
\mathscr{J}\left(D_{c 1}, \ldots, D_{c r}, E_{c 1}, \ldots, E_{c s}, Q\right) \triangleq \operatorname{tr} Q \tilde{R}
$$

subject to

$$
\begin{aligned}
& Q \in \mathbb{N}^{n}, \\
& 0=\tilde{A_{\alpha}} Q+Q \tilde{A_{\alpha}^{\mathrm{T}}}+\sum_{k=1}^{p} \gamma_{k} \tilde{A_{k}} Q \tilde{A}_{k}^{\mathrm{T}}+\tilde{V} .
\end{aligned}
$$

The relationship between the Auxiliary Minimization Problem and the Robust Stability and Performance Problem is straightforward as shown by the following observation.

Proposition 6.1. Suppose $\left(D_{c 1}, \ldots, D_{c r}, E_{c 1}, \ldots, E_{c s}, Q\right)$ satisfies (6.2), (6.3). Then

$$
\left(\tilde{A}+\Delta \tilde{A}, \tilde{V}^{1 / 2}\right) \text { is stabilizable for all variations in } \mathscr{U}
$$

if and only if $\tilde{A}+\Delta \tilde{A}$ is asymptotically stable for all variations in $\mathscr{U}$. In this case,

$$
J\left(D_{c 1}, \ldots, D_{c r}, E_{c 1}, \ldots, E_{c s}\right) \leq \mathscr{J}\left(D_{c 1}, \ldots, D_{c r}, E_{c 1}, \ldots, E_{c s}, Q\right) .
$$

Proof. With $\Omega$ given by (5.4), Proposition 5.1 implies that (4.3) is satisfied. Since (6.3) is a restatement of (4.4), the hypotheses of Theorem 4.1 are satisfied. Hence (6.4) is equivalent to robust stability and, in this case, performance bound (4.6) is guaranteed. Note that with definition (6.1), (6.5) is merely a restatement of (4.6).

The derivation of the necessary conditions for the Auxiliary Minimization Problem is based upon the Fritz John form of the Lagrange multiplier theorem. ${ }^{1}$ Application of this technique requires that $\left(D_{c 1}, \ldots, D_{c r}, E_{c 1}, \ldots, E_{c s}, Q\right)$ be restricted to the open set

$$
\mathscr{P} \triangleq\left\{\left(D_{c 1}, \ldots, D_{c r}, E_{c 1}, \ldots, E_{c s}, Q\right): Q \in \mathbb{P}^{n} \text { and } \mathscr{A} \text { is asymptotically stable }\right\},
$$

where

$$
\mathscr{A} \triangleq \tilde{A_{\alpha}} \oplus \tilde{A_{\alpha}}+\sum_{k=1}^{p} \gamma_{k} \tilde{A_{k}} \otimes \tilde{A_{k}} .
$$

The requirement $\left(D_{c 1}, \ldots, D_{c r}, E_{c 1}, \ldots, E_{c s}, Q\right) \in \mathscr{S}$ implies that $Q$ and its nonnegative-definite dual $P$ are unique solutions to the modified Lyapunov equations (6.3) and

$$
0=\tilde{A}_{\alpha}^{\mathrm{T}} P+P \tilde{A}_{\alpha}+\sum_{k=1}^{p} \gamma_{k} \tilde{A}_{k}^{\mathrm{T}} P \tilde{A_{k}}+\tilde{R}
$$

To obtain feedback gains an additional technical requirement is that $\left(D_{c 1}, \ldots, D_{c r}, E_{c 1}, \ldots, E_{c s}, Q\right)$ be confined to the open set

$$
\mathscr{S}^{+} \triangleq\left\{\left(D_{c 1}, \ldots, D_{c r}, E_{c 1}, \ldots, E_{c s}, Q\right) \in \mathscr{P}: \hat{C}_{i} Q \hat{C}_{i}^{\mathrm{T}}>0, i=1, \ldots, r \text {, and } \hat{B}_{j}^{\mathrm{T}} P \hat{B}_{j}>0, j=1, \ldots, s\right\} .
$$

The positive definiteness conditions in the definition of $\mathscr{S}^{+}$hold if $\hat{C}_{i}$ and $\hat{B}_{j}$ have full row and column rank, respectively, and $Q$ and $P$ are positive definite. As can be seen from the proof of Theorem 6.1 these conditions imply the existence of the projections $\nu_{i}$ and $\hat{\nu}_{j}$ corresponding to the two distinct types of feedback loops.

\footnotetext{
1 The Kuhn-Tucker theorem requires a priori verification of a constraint qualification which is difficult to confirm in the present context. The Fritz John version is less restrictive and hence more suitable.
} 
Remark 6.1. It is possible, of course, that the set $\mathscr{S}$ may be empty in which case our results do not apply. As will be seen, however, our approach does not require explicit verification that $\mathscr{S}$ be nonempty since robust stability is obtained as a consequence of robust performance. Indeed, the sets $\mathscr{S}$ and $\mathscr{S}^{+}$are for theoretical convenience only and need not be determined in practice. Furthermore, the constraint $\left(D_{c 1}, \ldots, D_{c r}, E_{c 1}, \ldots, E_{c s}, Q\right) \in \mathscr{S}$ is not required for either robust stability or robust performance since Proposition 6.1 shows that only (6.2)-(6.4) are needed. Rather, the set $\mathscr{S}$ constitutes sufficient conditions under which the Lagrange multiplier technique is applicable to the Auxiliary Minimization Problem.

Necessary conditions for the Auxiliary Minimization Problem can now be obtained.

Theorem 6.1. Suppose $\left(D_{c 1}, \ldots, D_{c r}, E_{c 1}, \ldots, E_{c s}, Q\right) \in \mathscr{S}^{+}$solves the Auxiliary Minimization Problem with $\mathscr{U}$ given by (5.1). Then the feedback gains $D_{c 1}, \ldots, D_{c r}, E_{c 1}, \ldots, E_{c s}$ are given by

$$
\begin{gathered}
D_{c i}=-R_{a i}^{-1} P_{a i} Q \hat{C}_{i}^{\mathrm{T}}\left(\hat{C}_{i} Q \hat{C}_{i}^{\mathrm{T}}\right)^{-1}, \quad i=1, \ldots, r, \\
E_{c j}=-\left(\hat{B}_{j}^{\mathrm{T}} P \hat{B}_{j}\right)^{-1} \hat{B}_{j}^{\mathrm{T}} P Q_{a j} V_{a j}^{-1}, \quad j=1, \ldots, s,
\end{gathered}
$$

where the $n \times n$ nonnegative-definite matrices $Q, P$ satisfy

$$
\begin{aligned}
& 0=\left(A_{\alpha}-\sum_{i=1}^{r} B_{i} R_{a i}^{-1} P_{a i} \nu_{i}\right) Q+Q\left(A_{\alpha}-\sum_{i=1}^{r} B_{i} R_{a i}^{-1} P_{a i} \nu_{i}\right)^{\mathrm{T}}+V_{0} \\
& +\sum_{k=1}^{p} \gamma_{k}\left(A_{k}-\sum_{i=1}^{r} B_{i k} R_{a i}^{-1} P_{a i} \nu_{i}\right) Q\left(A_{k}-\sum_{i=1}^{r} B_{i k} R_{a i}^{-1} P_{a i} \nu_{i}\right)^{\mathrm{T}} \\
& -\sum_{j=1}^{s} Q_{a j} V_{a j}^{-1} Q_{a j}^{\mathrm{T}}+\sum_{j=1}^{s} \hat{\nu}_{j \perp} Q_{a j} V_{a j}^{-1} Q_{a j}^{\mathbf{T}} \hat{\nu}_{j \perp}^{\mathrm{T}} \\
& 0=\left(A_{\alpha}-\sum_{j=1}^{s} \hat{\nu}_{j} Q_{a j} V_{a j}^{-1} C_{j}\right)^{\mathrm{T}} P+P\left(A_{\alpha}-\sum_{j=1}^{s} \hat{\nu}_{j} Q_{a j} V_{a j}^{-1} C_{j}\right)+R_{0} \\
& +\sum_{k=1}^{p} \gamma_{k}\left(A_{k}-\sum_{j=1}^{s} \hat{\nu}_{j} Q_{a j} V_{a j}^{-1} C_{j k}\right)^{\mathrm{T}} P\left(A_{k}-\sum_{j=1}^{s} \hat{v}_{j} Q_{a j} V_{a j}^{-1} C_{j k}\right) \\
& -\sum_{i=1}^{r} P_{a i}^{\mathrm{T}} R_{a i}^{-1} P_{a i}+\sum_{i=1}^{r} \nu_{i \perp}^{\mathrm{T}} P_{a i}^{\mathrm{T}} R_{a i}^{-1} P_{a i} \nu_{i \perp} \\
& \nu_{i} \triangleq Q \hat{C}_{i}^{\mathrm{T}}\left(\hat{C}_{i} Q \hat{C}_{i}^{\mathrm{T}}\right)^{-1} \hat{C}_{i}, \quad \nu_{i \perp} \triangleq I_{n}-\nu_{i}, \quad i=1, \ldots, r, \\
& \hat{\nu}_{j} \triangleq \hat{B}_{j}\left(\hat{B}_{j}^{\mathrm{T}} P \hat{B}_{j}\right)^{-1} \hat{B}_{j}^{\mathrm{T}} P, \quad \hat{\nu}_{j \perp} \triangleq I_{n}-\hat{\nu}_{j}, \quad j=1, \ldots, s .
\end{aligned}
$$

Furthermore, the auxiliary cost is given by

$$
\begin{aligned}
& \mathscr{J}\left(D_{c 1}, \ldots, D_{c r}, E_{c 1}, \ldots, E_{c s}, Q\right) \\
& \quad=\operatorname{tr}\left[Q\left(R_{0}+\sum_{i=1}^{p} \nu_{i}^{\mathrm{T}} P_{a i}^{\mathrm{T}} R_{a i}^{-1} P_{a i} \nu_{i}-2 R_{0 i} R_{a i}^{-1} P_{a i} \nu_{i}\right)\right] .
\end{aligned}
$$

Conversely if there exist $Q, P \in \mathbb{N}^{n}$ satisfying (6.9) and (6.10) then $Q$ satisfies (6.3) with $\left(D_{c 1}, \ldots, D_{c r}, E_{c 1}, \ldots, E_{c s}\right)$ given by (6.7) and (6.8), and $\mathscr{J}\left(D_{c 1}, \ldots, D_{c r}, E_{c 1}, \ldots, E_{c s}, Q\right)$ is given by (6.13).

Proof. To optimize (6.1) over the open set $\mathscr{S}^{+}$and subject to the constraint (6.3), form the Lagrangian

$$
\mathscr{L}\left(D_{c 1}, \ldots, D_{c r}, E_{c 1}, \ldots, E_{c s}, Q\right) \triangleq \operatorname{tr}\left[\lambda Q \tilde{R}+\left(\tilde{A} Q+Q \tilde{A}^{\mathrm{T}}+\sum_{k=1}^{p} \gamma_{k} \tilde{A}_{k} Q \tilde{A}_{k}^{\mathrm{T}}+\tilde{V}\right) P\right],
$$


where the Lagrange multipliers $\lambda \geq 0$ and $P \in \mathbb{R}^{n \times n}$ are not both zero. Setting $\partial \mathscr{L} / \partial Q=0, \lambda=0$ implies $P=0$ since $\mathscr{A}$ is asymptotically stable. Hence, without loss of generality set $\lambda=1$. Thus the stationarity conditions are given by

$$
\begin{aligned}
& \frac{\partial \mathscr{L}}{\partial Q}=\tilde{A}_{\alpha}^{\mathrm{T}} P+P \tilde{A}_{\alpha}+\sum_{k=1}^{P} \gamma_{k} \tilde{A}_{k}^{\mathrm{T}} P \tilde{A_{k}}+\tilde{R}=0, \\
& \frac{\partial \mathscr{L}}{\partial D_{c i}}=R_{a i} D_{c i} \hat{C}_{i} Q \hat{C}_{i}^{\mathrm{T}}+P_{a i} Q \hat{C}_{i}^{\mathrm{T}}=0, \quad i=1, \ldots, r, \\
& \frac{\partial \mathscr{L}}{\partial E_{c j}}=\hat{B}_{j}^{\mathrm{T}} P \hat{B}_{j} E_{c j} V_{a j}+\hat{B}_{j}^{\mathrm{T}} P Q_{a j}=0, \quad j=1, \ldots, s .
\end{aligned}
$$

Since $\left(D_{c l}, \ldots, D_{c r}, E_{c 1}, \ldots, E_{c s}, Q\right) \in \mathscr{S}^{+}, \hat{C}_{i} Q \hat{C}_{i}^{\mathrm{T}}$ and $\hat{B}_{j}^{\mathrm{T}} P \hat{B}_{j}$ are invertible and hence (6.15) and (6.16) imply (6.7) and (6.8). Finally, (6.9) and (6.10) are equivalent to (6.3) and (6.6).

Remark 6.2. Several special cases can be recovered from Theorem 6.1. For example, when the control weighting is nonsingular and the measurement noise is zero, i.e., when $\hat{u}_{i}$ and $\hat{y}_{i}$ are absent for $i=1, \ldots, r$, delete (6.8) and set $\hat{\nu}_{j}=0$ and $\hat{\nu}_{j \perp}=I_{n}$ in (6.9). In this case the last two terms in (6.9) cancel each other. Deleting also the uncertainty terms $A_{k}, B_{i k}, C_{j k}$ yields the results of [12] with the added features of correlated plant/measurement noise $\left(V_{0 j}\right)$ and cross weighting $\left(R_{0 i}\right)$. Furthermore, assuming a centralized structure for the static controller, i.e., $r=1$, yields the usual static output feedback result $[9,11]$.

We now combine Proposition 6.1 and Theorem 6.1 to obtain sufficient conditions for robust stability and robust performance.

Theorem 6.2. Suppose there exist $Q, P \in \mathbb{N}^{n}$ satisfying (6.9) and (6.10) and let the feedback gains $\left(D_{c 1}, \ldots, D_{c r}, E_{c 1}, \ldots, E_{c s}\right)$ be given by (6.7) and (6.8). Then $\left(\tilde{A}+\Delta \tilde{A}, \tilde{V}^{1 / 2}\right)$ is stabilizable for all variations in $\mathscr{U}$ if and only if $\tilde{A}+\Delta \tilde{A}$ is asymptotically stable for all variations in $\mathscr{U}$. In this case the performance (3.6) of the closed-loop system satisfies the bound

$$
J\left(D_{c 1}, \ldots, D_{c r}, E_{c 1}, \ldots, E_{c s}\right) \leq \operatorname{tr}\left[Q\left(R_{0}+\sum_{i=1}^{p} \nu_{i}^{\mathrm{T}} P_{a i}^{\mathrm{T}} R_{a i}^{-1} R_{i} R_{a i}^{-1} P_{a i} \nu_{i}-2 R_{0 i} R_{a i}^{-1} P_{a i} \nu_{i}\right)\right] .
$$

Proof. The converse portion of Theorem 6.1 shows that $Q$ satisfies (6.3) with $\left(D_{c 1}, \ldots, D_{c r}, E_{c 1}, \ldots, E_{c s}\right)$ given by (6.7) and (6.8). Hence, with the stabilizability assumption (6.4), Proposition 6.1 implies robust stability and performance.

\section{Decentralized design algorithm}

To illustrate the preceding theoretical development it is worthwhile outlining the steps involved in applying the results to the decentralized design problem. These steps constitute the following algorithm for designing robust decentralized static output feedback controllers.

Algorithm 7.1. To apply Theorem 6.2, carry out the following steps:

Step 1. Specify a decentralized feedback structure of the form (3.4), (3.5).

Step 2. Specify performance weights $R_{0}, R_{0 i}$ and $R_{i}$ as well as noise intensities $V_{0}, V_{0 j}$, and $V_{j}$.

Step 3. Specify the uncertainty set $\mathscr{U}$ in terms of the uncertainty structure matrices $A_{k}, B_{i k}, C_{j k}$ and bounds $\alpha_{k}$.

Step 4. Choose $\alpha>0$.

Step 5. Numerically solve the design equations (6.9) and (6.10). 
Step 6. Evaluate the feedback gains (6.7) and (6.8).

Step 7. Verify that $\left(\tilde{A}+\Delta \tilde{A}, \tilde{V}^{1 / 2}\right)$ is stabilizable for all variations in $\mathscr{U}$ or, equivalently, that $\tilde{A}+\Delta \tilde{A}$ is asymptotically stable for all variations in $\mathscr{U}$.

Step 8. Evaluate the robust performance bound (6.17).

We note that in certain cases Step 7 can be omitted. For example, if $V_{0}$ is positive definite then so is $\tilde{V}$ and $\left(\tilde{A}+\Delta \tilde{A}, \tilde{V}^{1 / 2}\right)$ is controllable for all $\Delta \tilde{A}$ (see Remark 4.2). Step 7 essentially ensures that the closed-loop system does not possess hidden unstable modes.

The crucial step in Algorithm 7.1 is, of course, the numerical solution of (6.9), (6.10) in Step 5. For the decentralized problem in the absence of parameter uncertainty, the numerical solution of (6.9), (6.10) was considered in [12] where several iterative algorithms were developed. When parameter uncertainty bounds are present, the additional terms involving $Q$ and $P$ in (6.9) and (6.10) must be included in the iteration. No additional difficulty is entailed, however, since the linearity of these terms permits a Newton-Kleinman type iterative schem [7].

If a numerical solution cannot be obtained in Step 5 then the problem data can be modified. This can be done systematically by means of (1) changing the arbitrary constant parameter $\alpha$ in Step 4; (2) decreasing the bounds $\alpha_{k}$ and/or modifying the uncertainty structure matrices $A_{k}, B_{i k}$, and $C_{j k}$ in Step 3; (3) modifying the performance weightings and noise intensities in Step 2; and, finally, altering the decentralized controller structure in Step 1.

\section{References}

[1] D.S. Bernstein, Robust static and dynamic output-feedback stabilization: Deterministic and stochastic perspectives, IEEE Trans. Automat. Control. 32 (1987) 1076-1084.

[2] D.S. Bernstein and W.M. Haddad, Robust stability and performance analysis for state space systems via quadratic Lyapunov Bounds, Proc. IEEE Conf. Decision and Control, Austin, TX (Dec. 1988).

[3] J.W. Brewer, Kronecker products and matrix calculus in system theory, IEEE Trans. Circuits and Systems 25 (1978) $772-781$.

[4] S.S.L. Chang and T.K.C. Peng, Adaptive guaranteed cost control of systems with uncertain parameters, IEEE Trans. Automat. Control. 17 (1972) 474-483.

[5] D.C. Hyland and D.S. Bernstein, The optimal projection equations for fixed-order dynamic compensation, IEEE Trans. Automat. Control. 29 (1984) 1034-1037.

[6] P.T. Kabamba, R.W. Longman and S. Jian-Guo, A homotopy approach to the feedback stabilization of linear systems, $J$. Guidance Control Dynamics 10 (1987) 422-432.

[7] D.L. Kleinman, On an iterative technique for the Riccati equation computation, IEEE Trans. Automat. Control. 13 (1968) 114-115.

[8] O.I. Kosmidou and P. Bertrand, Robust-controller design for systems with large parameter variations, Internat. J. Control 45 (1987) 927-938.

[9] W.S. Levine and M. Athans, On the determination of the optimal constant output feedback gains for linear multivariable systems, IEEE Trans. Automat. Control. 15 (1970) 44-48.

[10] M. Mariton and R. Bertrand, A homotopy algorithm for solving coupled Riccati equations, Optim. Control. Appl. Meth. 6 (1985) 351-357.

[11] J. Medanic, On stabilization and optimization by output feedback, Proc. Twelfth Asilomar Conf. Circuits Systems Comput. (1978) 412-416.

[12] S. Renjen and D.P. Looze, Synthesis of decentralized output state regulators, Proc. Amer. Contr. Conf., Arlington, VA (1982) $758-762$.

[13] S. Richter, A homotopy algorithm for solving the optimal projection equations for fixed-order dynamic compensation: Existence, convergence and global optimality, Proc. Amer. Control. Conf., Minneapolis, MN (June 1987) 1527-1531.

[14] W.M. Wonham, Linear Multivariable Control: A Geometric Approach (Springer-Verlag, New York, 1979). 